\title{
Lectin-type oxidized LDL receptor 1 modulates matrix metalloproteinase 2 production in peri-implantitis
}

\author{
QIAN ZHANG ${ }^{1,2}$, TAO XU $^{2,3}$, NA BAI $^{1,2}$, FEI TAN $^{1,2}$, HONGMEI ZHAO $^{1,2}$ and JIE LIU ${ }^{1,2}$ \\ ${ }^{1}$ Department of Prosthodontics, The Affiliated Hospital of Qingdao University; \\ ${ }^{2}$ School of Stomatology of Qingdao University; ${ }^{3}$ Department of Orthodontics, \\ The Affiliated Hospital of Qingdao University, Qingdao, Shandong 266000, P.R. China
}

Received June 16, 2021; Accepted September 27, 2021

DOI: $10.3892 / \mathrm{etm} .2021 .11094$

\begin{abstract}
Peri-implantitis is a disease in which inflammatory lesions that affect the surrounding soft and hard tissues develop. Matrix metalloproteinase 2 (MMP2) is hypothesized to be involved in this destructive process. However, the regulatory mechanism of action of MMP2 in the peripheral tissues of the implant are not fully understood. To determine the expression of MMP2 in peri-implantitis, peri-implant crevicular fluid (PICF) was collected from patients with peri-implantitis. The healthy volunteers' peripheral blood human macrophages infected with Porphyromonas gingivalis ( $P$. gingivalis) were used as a cell model to explore the regulatory mechanism of MMP2 regarding dental implants. Western blotting, reverse transcriptionquantitative PCR and immunofluorescence staining were used to measure the expression of MMP2 in the present study. MMP2 expression was increased in the PICF of the patients with peri-implantitis and in human macrophages infected with $P$. gingivalis. Lectin-type oxidized LDL receptor 1 (LOX-1) mediated the expression of MMP2 in human macrophages upon infection of $P$. gingivalis, whereas dendritic cell-associated c-type lectin-1 did not appear to be involved in this regulatory process. However, JNK and ERK1/2 were involved in $P$. gingivalis induced MMP2 expression. The results of this study showed that MMP2 was involved in peri-implantitis. MMP2 was upregulated by LOX-1 in a JNK and ERKk1/2 signaling dependent manner in the cell model. The LOX-1/MMP2 signaling pathway may thus be a potential target for management of peri-implantitis.
\end{abstract}

\section{Introduction}

Dental implants have become an increasingly popular choice of treatment for replacing missing teeth (1). Peri-implantitis

Correspondence to: Dr Jie Liu, Department of Prosthodontics, The Affiliated Hospital of Qingdao University, 16 Jiangsu Road, Qingdao, Shandong 266000, P.R. China

E-mail: dentistqianzhang@126.com

Key words: peri-implantitis, Porphyromonas gingivalis, matrix metalloproteinase 2, lectin-type oxidized LDL receptor 1 is the development of serious inflammatory lesions following dental implant therapy. The surrounding hard and soft tissues are affected in peri-implantitis, which can lead to the loss of the implant (2). Previous studies of these therapeutic strategies have not been able to identify a single treatment regimen that is suitably effective against peri-implantitis (3-5).

Host inflammatory cytokines and osteo-immunoinflammatory mediators can lead to the loss of supporting tissues around the implant (4). Matrix metalloproteinases (MMPs) are important for bone repair, development and inflammation. As one of the collagenases, matrix metalloproteinase 2 (MMP2) produced by inflamed cells is involved in several inflammatory diseases (6). In murine arthritis induced by group B streptococci, MMP2 is involved in the production of pro-inflammatory cytokines and the degradation of extracellular matrix components (7). In human astroglia, the ERKk1/2-NF- $\mathrm{kB}$ signaling pathway mediates Toxoplasma gondii induced MMP2 production to cleave fibronectin (8). In apical periodontitis, the upregulation of MMP2 and MMP9 is modulated by the TLR2MyD88 signaling pathway (9).

A previous study showed that MMP2 was upregulated when stimulated by Porphyromonas gingivalis ( $P$. gingivalis) lipopolysaccharide (PG-LPS) in THP-1 macrophages (10). However, the regulatory mechanism and signal transduction pathway by which MMP2 exerts its effects in patients who have not received implants have not been assessed previously, to the best of our knowledge. The aim of the present study was to characterize the regulatory mechanism of MMP2 in peri-implantitis.

\section{Materials and methods}

Peri-implant crevicular fluid (PICF) collection. In the present study, 10 cases of healthy implants (age, $45 \pm 4.6$ years; sex, five males and five females) and 10 cases of peri-implantitis implants (age, $46 \pm 4.4$ years; sex, five males and five females) were studied. Each patient had $\geq$ two dental implants, and both implants were investigated. The diagnostic criteria, exclusion criteria and inclusion criteria have been described previously used in our preliminary work (10). Sterile Periopapers (Oraflow) were used to collect PICF samples, which were then placed on the gingival sulcus and implant for $30 \mathrm{sec}$. For further testing, $50 \mathrm{mM}$ phosphate buffer containing $0.1 \mathrm{mM}$ 
phenylmethylsulfonyl fluoroether was used to elute the PICF contents.

This study was performed in accordance with the Declaration of Helsinki and was approved by the Ethics Committee of The Hospital Affiliated to Qingdao University (approval no. 3/6/2018 \#20180306; Qingdao, China). All patients provided written informed consent (1).

Isolation of human macrophages. Human macrophages were obtained from the peripheral blood of healthy volunteers after receiving approval from the Ethics Committee of The Affiliated Hospital of Qingdao University. The mononuclear fraction was isolated by density gradient centrifugation at $400 \mathrm{x} \mathrm{g}$ for $30 \mathrm{~min}$ at room temperature using Ficoll Paque Plus according to the manufacturer's protocol (TBD Biotech). After washing with PBS at room temperature, cells were centrifuged at $200 \mathrm{x}$ g for $5 \mathrm{~min}$ at room temperature and suspended in RPMI-1640 media (Hyclone; Cytiva) at a density of $1 \times 10^{6}$ cells $/ \mathrm{ml}$. Cells were confirmed to be macrophages (macrophages $>95 \%$ ) by Diff-Quik (cat. no. G1541; Beijing Solarbio Science \& Technology Co., Ltd.) stain (Diff-Quik I for $10 \mathrm{sec}$ and Diff-Quik II for $15 \mathrm{sec}$ at room temperature).

Bacterial culture. P. gingivalis, provided by the Oral Laboratory of the Hospital affiliated to Qingdao University, were grown overnight in an incubator at $37^{\circ} \mathrm{C}$ under anaerobic conditions $\left(90 \% \mathrm{~N}\right.$ and $5 \% \mathrm{CO}_{2}$ and $\left.25 \% \mathrm{H}_{2}\right)$ in gifu anaerobic medium (cat. no. LA4500; Beijing Solarbio Science \& Technology Co., Ltd.) broth with an optical density of $650 \mathrm{~nm}$ of $1.0\left(1 \times 10^{9}\right.$ colony forming units $\left./ \mathrm{ml}\right)(11)$. The $P$. gingivalis was added to RPMI-1640 media to infect the human macrophages after washing the cells twice by RPMI-1640. The multiplicity of infection (MOI) used was 20.

MMP2 expression in human macrophages infected with $P$. gingivalis. Western blotting and reverse transcriptionquantitative (RT-q)PCR were used to detect MMP2 expression in cells treated with live $P$. gingivalis bacteria after $0,1 / 4,1 / 2$, 1, 4 and $16 \mathrm{~h}$, to measure the expression of MMP2 in human macrophages infected with $P$. gingivalis.

To reveal the role of dectin-1 and LOX-1 in the production of MMP2 following infection with $P$. gingivalis bacteria, dectin-1 (cat. no. AF1859) or LOX-1 (cat. no. MAB1798) neutralizing antibodies (10 $\mu \mathrm{g} / \mathrm{ml}$; R\&D Systems, Inc.) (12) and the LOX-1 inhibitor, polyinosinic acid (Poly I; Sigma-Aldrich; Merck $\mathrm{KGaA}$; $0.25 \mathrm{mg} / \mathrm{ml}$ ) (13) or a dectin-1 inhibitor, laminarin (Sigma-Aldrich; Merck KGaA; $0.3 \mathrm{mg} / \mathrm{ml}$ ) (1) were used to pretreat human macrophages for $2 \mathrm{~h}$ at room temperature, which were then treated with $P$. gingivalis bacteria $2 \mathrm{~h}$ later at room temperature.

To investigate the effects of Erk1/2 and JNK on MMP2 production with $P$. gingivalis, human macrophages were pretreated with the JNK inhibitor, SP600125 (Selleck Chemicals; $40 \mu \mathrm{M}$ ) and the Erk1/2 inhibitor, U0126 (Selleck Chemicals; $20 \mu \mathrm{M})(1), 2 \mathrm{~h}$ at room temperature before treatment with live $P$. gingivalis bacteria at room temperature. The controls were DMSO, sterile water and goat IgG $(10 \mu \mathrm{g} / \mathrm{ml}$; cat. no. AB-108-C; R\&D Systems, Inc.). Live $P$. gingivalis were used to treat human macrophages for 0 and $16 \mathrm{~h}$ at room temperature for Western blot analysis and for 0 and $4 \mathrm{~h}$ at room temperature at a MOI of 20 for RT-qPCR.

Western blotting. Total proteins from human macrophages were lysed by RIPA Lysis Buffer (Beijing Solarbio Science \& Technology Co., Ltd.). The total protein contents were quantified using a bicinchoninic acid assay (Beyotime Institute of Biotechnology). Protein was loaded into each well $(60 \mu \mathrm{g})$ of a 10\% SDS-gel (Bio-Rad Laboratories, Inc.), resolved by SDS-PAGE and transferred to a PVDF membrane (EMD Millipore). The membranes were put in blocking buffer (cat. no. P0023B; Beyotime Institute of Biotechnology) for $2 \mathrm{~h}$. The membranes were incubated overnight with an anti- $\beta$-actin (1:1,000 Cell Signaling Technologies, Inc.) or anti-MMP2 (1:500 ProteinTech Group, Inc.) primary antibodies at $4^{\circ} \mathrm{C}$, followed by incubation with the corresponding HRP-tagged secondary antibodies at room temperature for $2 \mathrm{~h}(1: 1,000$ cat. nos. 7074 and 7076; Cell Signaling Technologies, Inc.). Clarity Western ECL Substrate (cat. no. 1705061; Bio-Rad Laboratories, Inc.) combined with the Fusion Solo System (FUSION-FX7 advanced; Vilber Lourmat) was used to visualize signals (ImageJ 1.48; National Institutes of Health).

Immunofluorescence staining. MMP2 immunofluorescence was used to stain small tissue fragments of superficial gingiva in patients with peri-implantitis. The immunofluorescence protocol was performed as described previously (10). Anti-MMP2 antibody (1:50; ProteinTech Group, Inc.) was used as the primary antibody overnight at $4^{\circ} \mathrm{C}$, and an Alexa Fluor 488-conjugated goat anti-rabbit IgG (1:1,000; cat. no. 4412, Cell Signaling Technology, Inc.) was used as the secondary antibody at room temperature for $1 \mathrm{~h}$, followed by DAPI staining at room temperature for $10 \mathrm{~min}(20 \mu \mathrm{g} / \mathrm{ml}$; cat. no. 4083; Cell Signaling Technology, Inc.). Images were captured using a fluorescence microscope (magnification, $\mathrm{x} 400$ ).

$R T$-qPCR. RT-qPCR was performed as described previously (10). At a pre-set time, the cell culture medium was carefully removed before $500 \mu 1$ RNAiso Plus (cat. no. 9109; Takara Bio, Inc.) was added into the cell culture plate followed by incubation at room temperature for $5 \mathrm{~min}$ to facilitate cell lysis. cDNA template for PCR was prepared from RNA $(2 \mu \mathrm{g})$ from human macrophages by reverse transcription (cat. no. RR066A; PrimeScript ${ }^{\mathrm{TM}}$ RT-PCR Kit Perfect Real Time; Takara Bio, Inc.). The reaction temperature of reverse transcription is $37^{\circ} \mathrm{C}$ for $15 \mathrm{~min}, 85^{\circ} \mathrm{C}$ for $5 \mathrm{sec}$. DEPC-treated water was used to dilute cDNA products 1:25. PCR was assayed in $20 \mu \mathrm{l}$ reaction system consisting of $2 \mu \mathrm{l}$ of $1 \mu \mathrm{g}$ diluted cDNA (1:25), $10 \mu$ l of TB Green ${ }^{\circledR}$ Premix Ex Taq ${ }^{\mathrm{TM}}$ (cat. no. RR420A; Takara Bio, Inc.), $1 \mu \mathrm{l} 100 \mu \mathrm{M}$ diluted primers (1:9) and $7 \mu \mathrm{l}$ DEPC-treated water) for $\mathrm{qPCR}$. All reactions were performed with the following cycling parameters: $95^{\circ} \mathrm{C}$ for $30 \mathrm{sec}$, followed by 40 cycles of $95^{\circ} \mathrm{C}$ for $5 \mathrm{sec}, 60^{\circ} \mathrm{C}$ for $30 \mathrm{sec}$ and a final stage of $95^{\circ} \mathrm{C}$ for $15 \mathrm{sec}, 60^{\circ} \mathrm{C}$ for $30 \mathrm{sec}$ and $95^{\circ} \mathrm{C}$ for $15 \mathrm{sec}$. The primer pair sequences were: $\beta$-actin forward, $5^{\prime}$-TGGCACCCA GCACAATGAA-3' and reverse, 5'-CTAAGTCATAGTCCG CCTAGAAGCA-3' and MMP2 forward, 5'-AGTTTCCAT TCCGCTTCCAG-3' and reverse, 5'-CGGTCGTAGTCCTCA GTGGT-3'. $\beta$-actin was used as the loading control. Data were quantified using the $2^{-\Delta \Delta \mathrm{Cq}}$ method (14). 


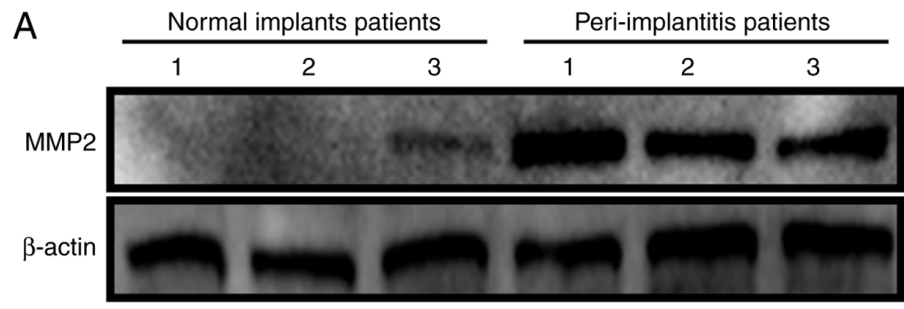

B

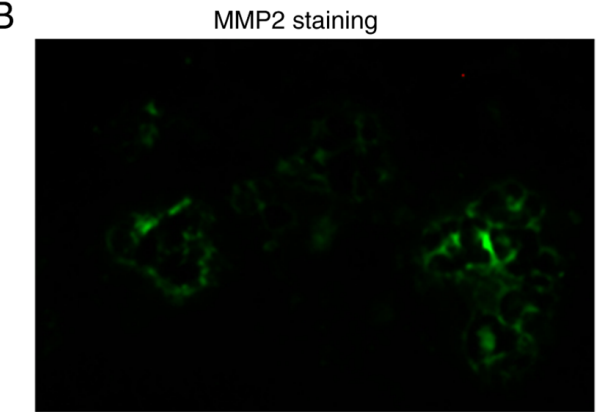

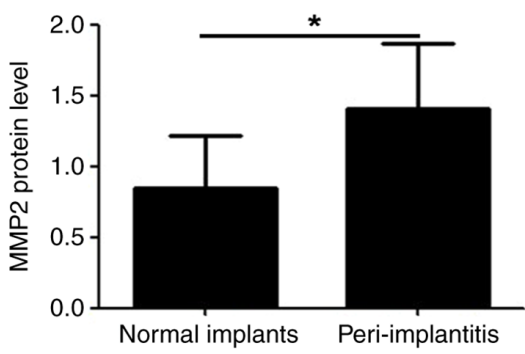

DAPI

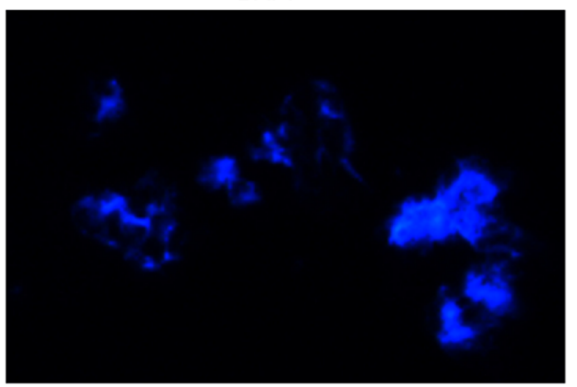

Figure 1. MMP2 expression is increased in patients with peri-implantitis. (A) In patients with peri-implant inflammation (n=10/group), the protein levels of MMP2 in PICF were higher than that in healthy patients. (B) Immunofluorescence staining showed expression of MMP2 (green) in the peri-implantitis patients' gingival surface (magnification, $\mathrm{x} 400$ ). " $\mathrm{P}<0.05$. MMP2, matrix metalloproteinase 2; PICF, peri-implant crevicular fluid.

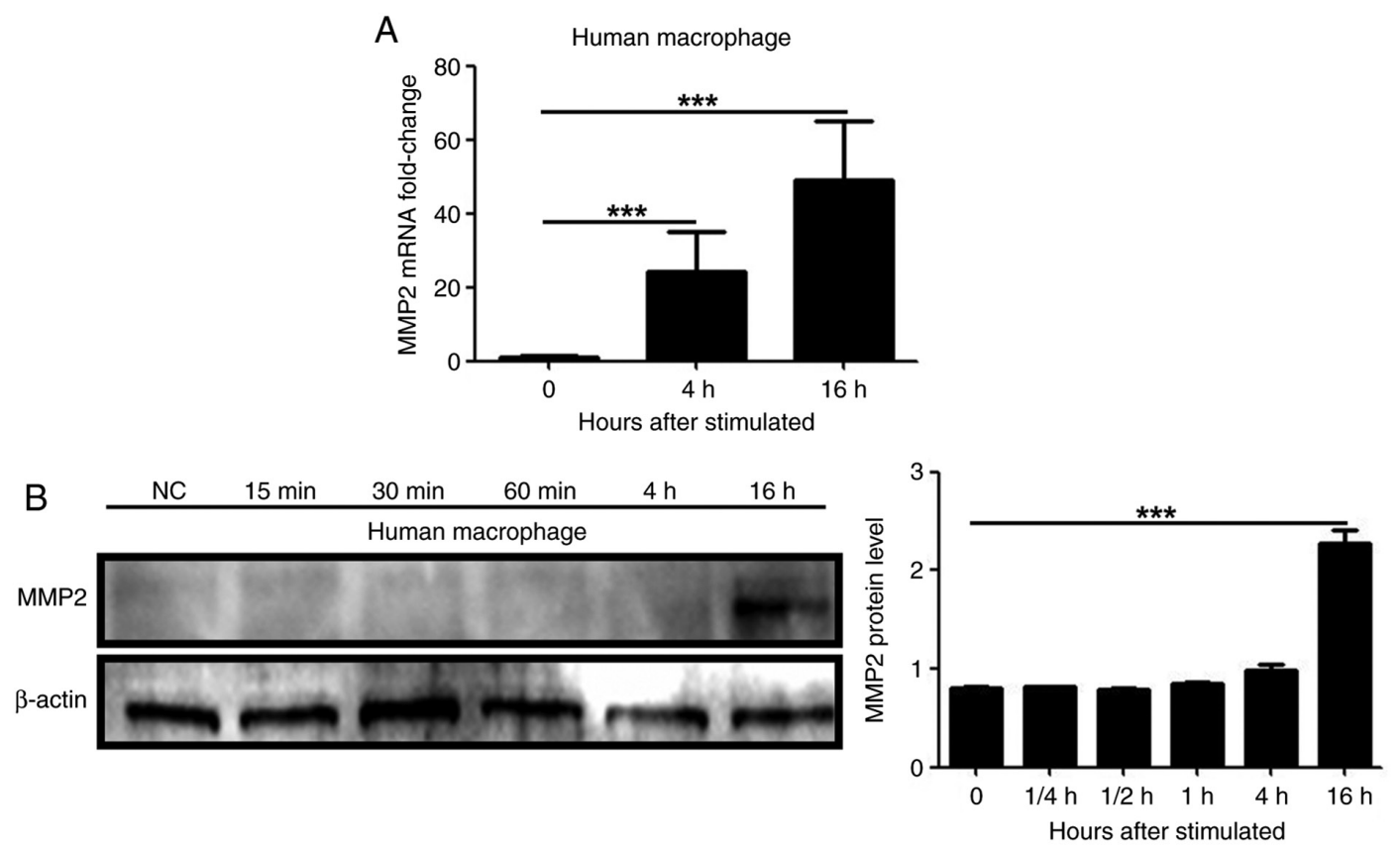

Figure 2. MMP2 expression is increased in $P$. gingivalis infected human macrophages. (A) MMP2 mRNA expression was elevated 4 and $16 \mathrm{~h}$ after infection of human macrophages with P. gingivalis. (B) MMP2 protein levels were higher $16 \mathrm{~h}$ after infection with $P$. gingivalis bacteria. ${ }^{* * *} \mathrm{P}<0.001$. MMP2, matrix metalloproteinase 2; P. gingivalis/P. g, Porphyromonas gingivalis; NC, negative control.

Statistical analysis. One-way ANOVA followed by a post-hoc Tukey's test was used to compare the differences between groups with SPSS 19.0 (IBM Corp.), which was presented as the mean $\pm \mathrm{SD}$ from three experimental repeats. $\mathrm{P}<0.05$ was considered to indicate a statistically significant difference.

\section{Results}

MMP2 expression is increased in peri-implantitis. Compared to healthy implants, the MMP2 protein expression levels were higher in the PICF of patients with peri-implantitis $(\mathrm{P}<0.05$; Fig. 1A). The superficial staining of peri-implantitis patients around implants also showed expression of MMP2 (green) (Fig. 1B). The results demonstrated that MMP2 may be involved in the pathogenesis of dental peri-implantitis.

MMP2 expression is increased in P. gingivalis infected human macrophages. MMP2 mRNA expression was increased after 4 and $16 \mathrm{~h}$ following infection of human macrophages with live $P$. gingivalis bacteria $(\mathrm{P}<0.01 ; \mathrm{Fig}$. $2 \mathrm{~A})$. MMP 2 production 


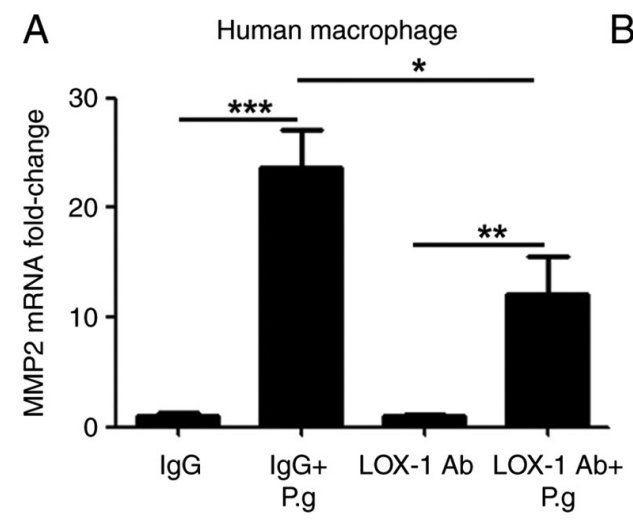

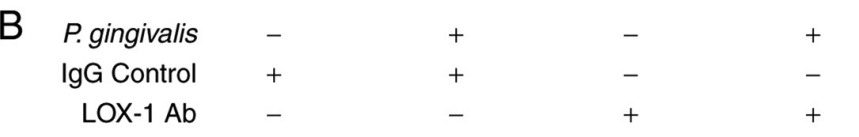
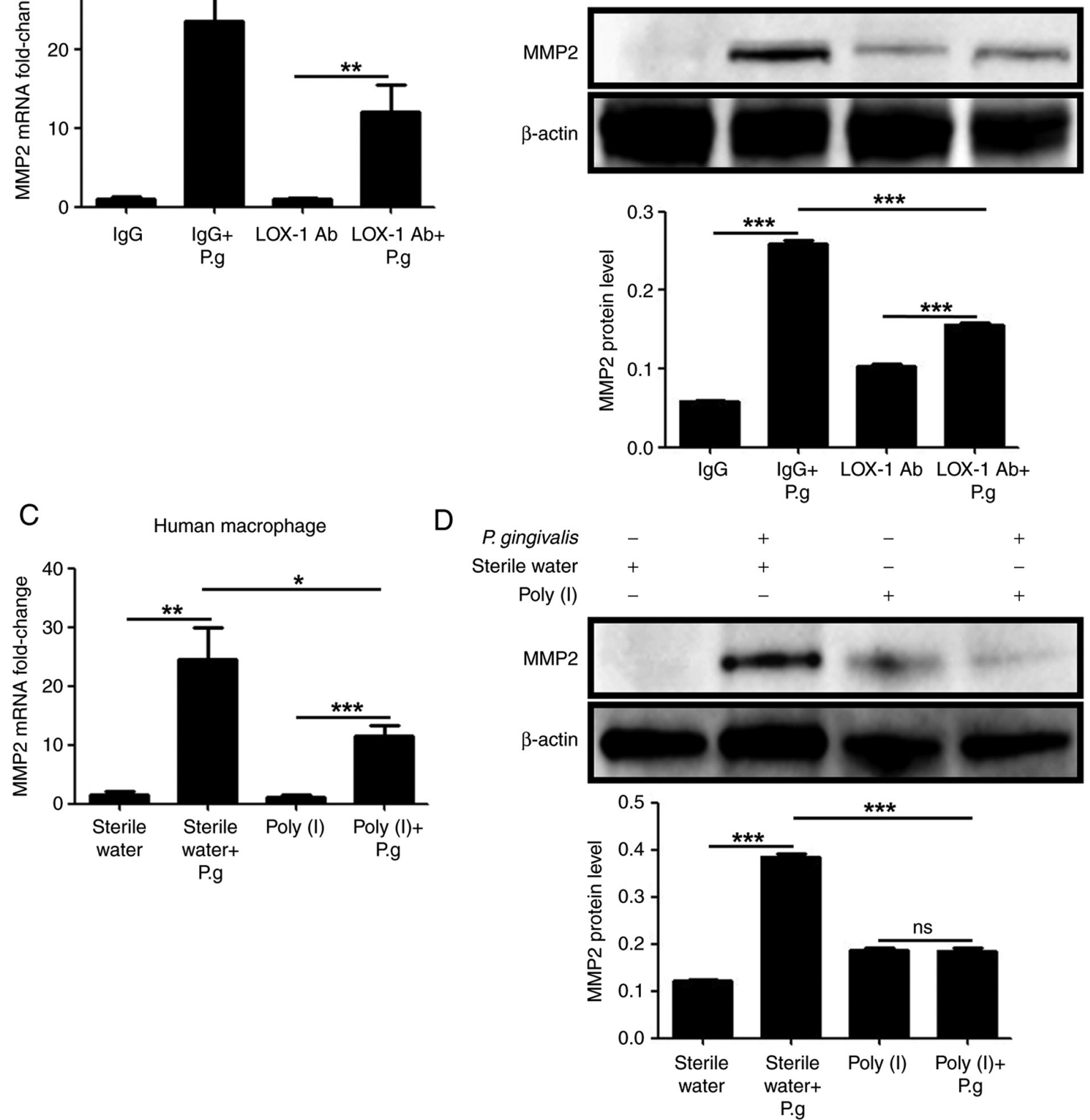

Figure 3. MMP2 production in P. gingivalis infected human macrophages is mediated by LOX-1. The MMP2 (A) mRNA and (B) protein expression levels were lower when pre-treated with the LOX-1 neutralizing antibody. The MMP2 (C) mRNA and (D) protein levels were lower when pre-treated with the LOX-1 inhibitor. ${ }^{*} \mathrm{P}<0.05,{ }^{* *} \mathrm{P}<0.01,{ }^{* * * *} \mathrm{P}<0.001$. ns, not significant; MMP2, matrix metalloproteinase 2; P. gingivalis, Porphyromonas gingivalis; LOX-1, Lectin-type oxidized LDL receptor 1; IgG, immunoglobulin; Poly (I), polyinosinic acid; Ab, antibody.

also increased at the protein level $16 \mathrm{~h}$ after infection with $P$. gingivalis bacteria $(\mathrm{P}<0.01$; Fig. $2 \mathrm{~B})$. These results demonstrated that MMP2 production in human macrophages could be induced by $P$. gingivalis.

MMP2 production in $P$. gingivalis infected human macrophages is mediated by LOX-1. To study the function of LOX-1 in human macrophages in the production of MMP2 in cells infected with $P$. gingivalis, LOX-1 expression was decreased using neutralizing antibodies or inhibitors. The increase in MMP2 expression in infected macrophages at the mRNA $(\mathrm{P}<0.05$; Fig. $3 \mathrm{~A}$ and $\mathrm{C})$ and protein $(\mathrm{P}<0.01$; Fig. 3B and D) levels were decreased by the LOX-1 neutralizing antibody or inhibitor pre-treatments. These results demonstrated that LOX-1 mediated MMP2 production induced by the infection of human macrophages with live $P$. gingivalis bacteria.

MMP2 production in $P$. gingivalis infected human macrophages is independent of dectin-1. To determine the function of dectin-1 in the MMP2 production in human macrophages infected with live $P$. gingivalis bacteria, neutralizing antibodies and inhibitors were used to inhibit dectin-1 activity. The MMP2 mRNA (P>0.05; Fig. 4A and C) and protein $(\mathrm{P}>0.05$; Fig. 4B and $\mathrm{D}$ ) levels did not differ significantly between treatment with the dectin-1 neutralizing 

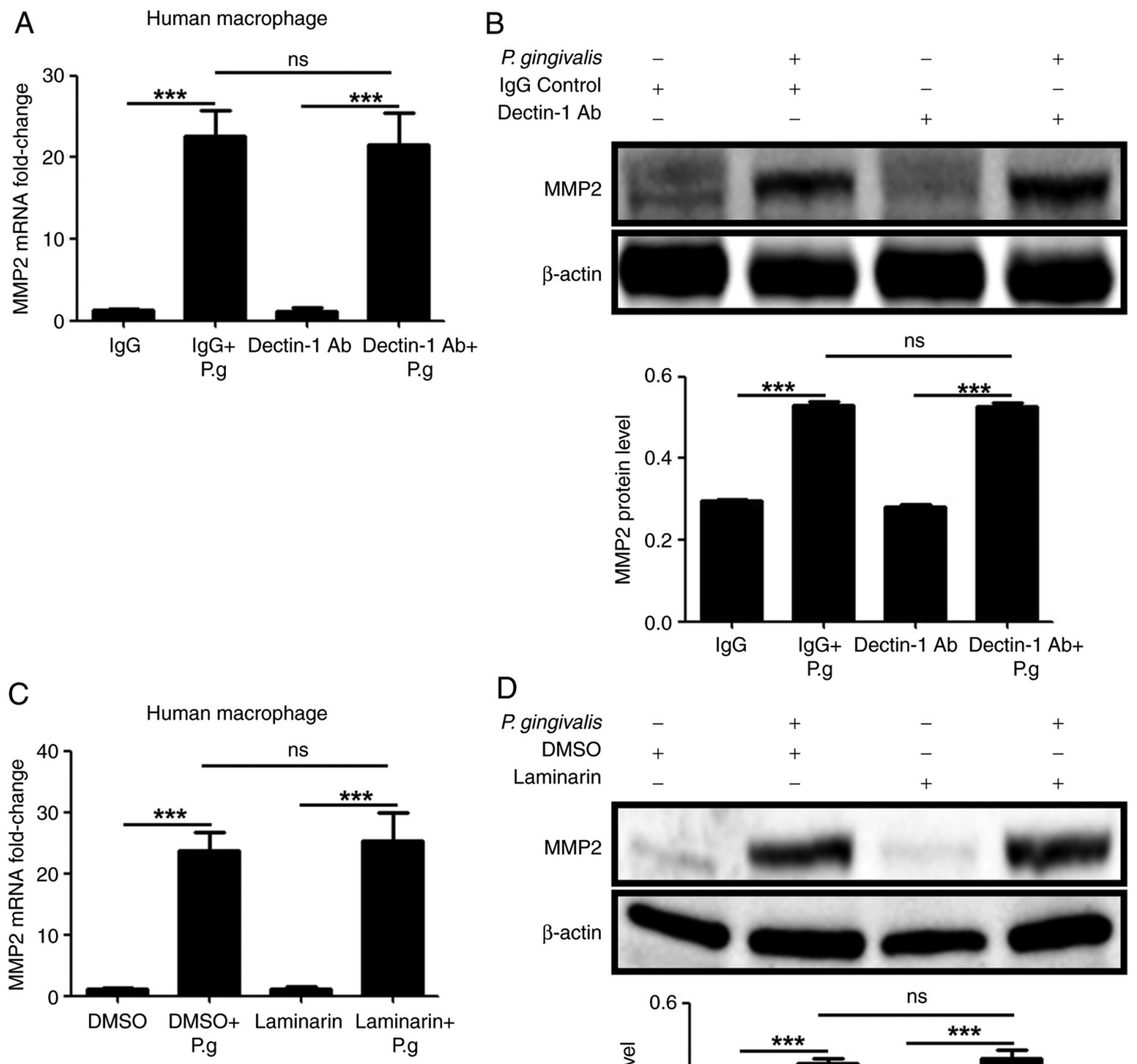

$\mathrm{D}$
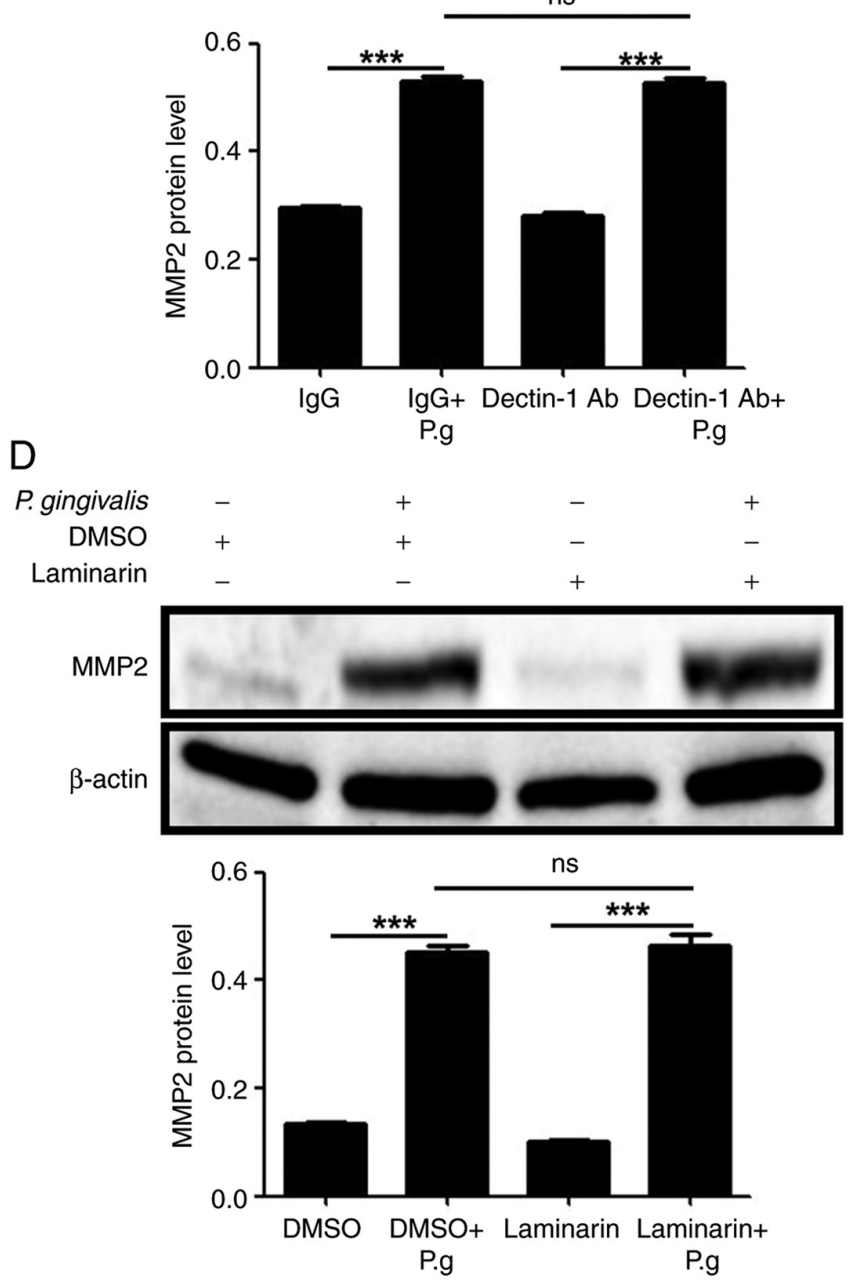

Figure 4. MMP2 production in P. gingivalis infected human macrophages was independent of dectin-1. Pre-treatment with dectin-1 neutralizing antibody did not significantly affect the (A) mRNA and (B) protein expression levels of MMP2. Similarly, the MMP2 (C) mRNA and (D) protein expression levels were not significantly affected with dectin-1 inhibitor pre-treatment either. ${ }^{* * * *} \mathrm{P}<0.001$. ns, not significant; MMP2, matrix metalloproteinase 2; P. gingivalis, Porphyromonas gingivalis; IgG, immunoglobulin; Ab, antibody.

antibody or inhibitor pre-treatment compared to the control untreated cells. These results showed that MMP2 production was independent of dectin- 1 in the macrophages infected with live $P$. gingivalis bacteria.

$J N K$ and Erk1/2 are responsible for $P$. gingivalis induced $M M P 2$ production. JNK and Erk1/2 inhibitors were used to pretreat the human macrophages to investigate their role in MMP2 production following $P$. gingivalis infection. The results indicated that the upregulated MMP2 mRNA $(\mathrm{P}<0.05$; Fig. 5A and C) and protein $(\mathrm{P}<0.01$; Fig. 5B and D) levels, when infected with live $P$. gingivalis bacteria, were decreased by JNK or Erk1/2 inhibition. These results demonstrated that LOX-1 upregulated MMP2 with the infection of $P$. gingivalis bacteria was mediated by Erk1/2.

\section{Discussion}

As one of the collagenases, MMP2 mediates multiple functions associated with oral inflammation, bone development and repair. Gonçalves et al (15) found that the levels of MMP2, MMP3, MMP8, MMP9, MMP12 and MMP13 in gingival crevicular fluid were significantly correlated with the severity of localized aggressive periodontitis. da Costa et al (16) 

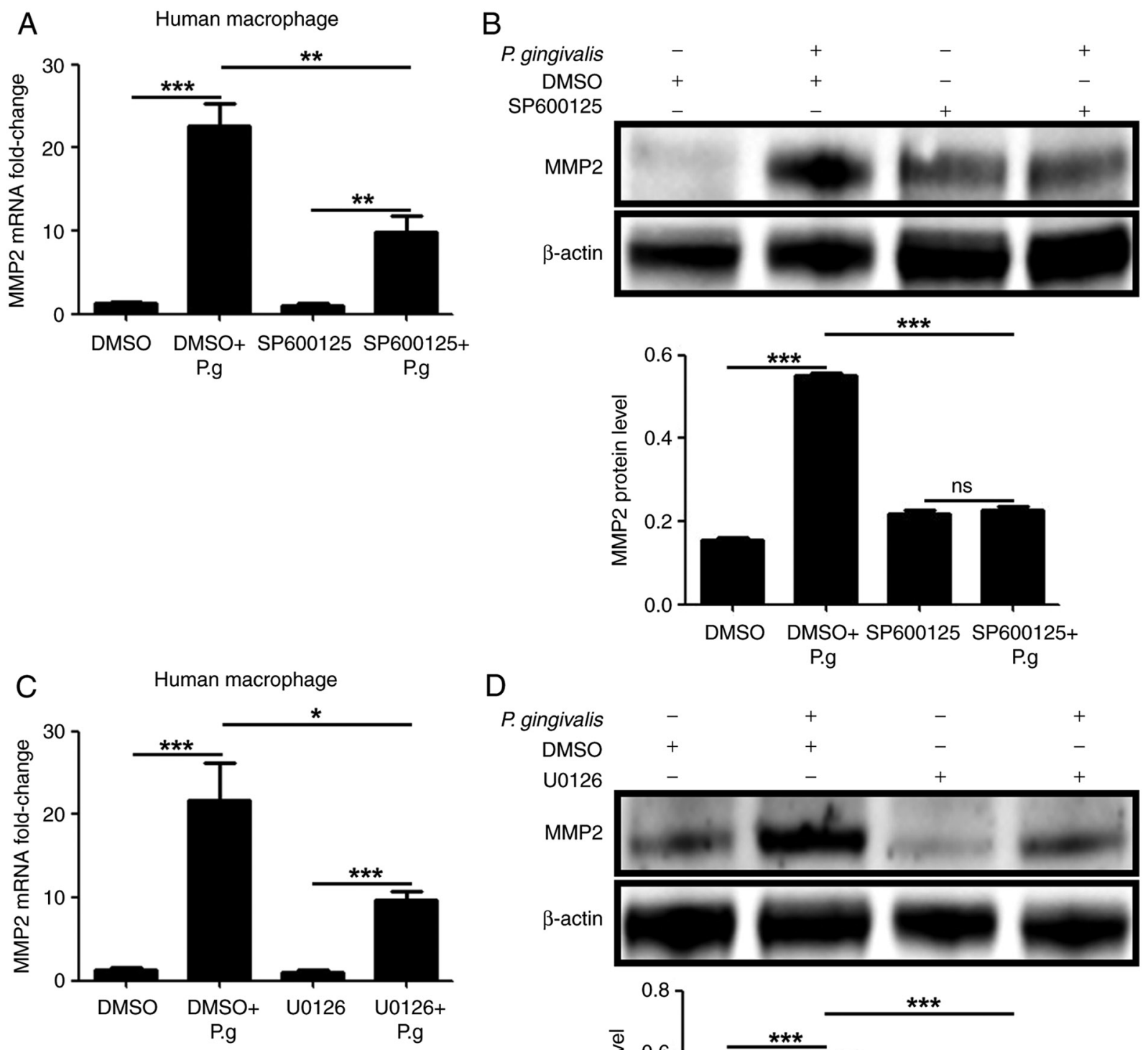

D
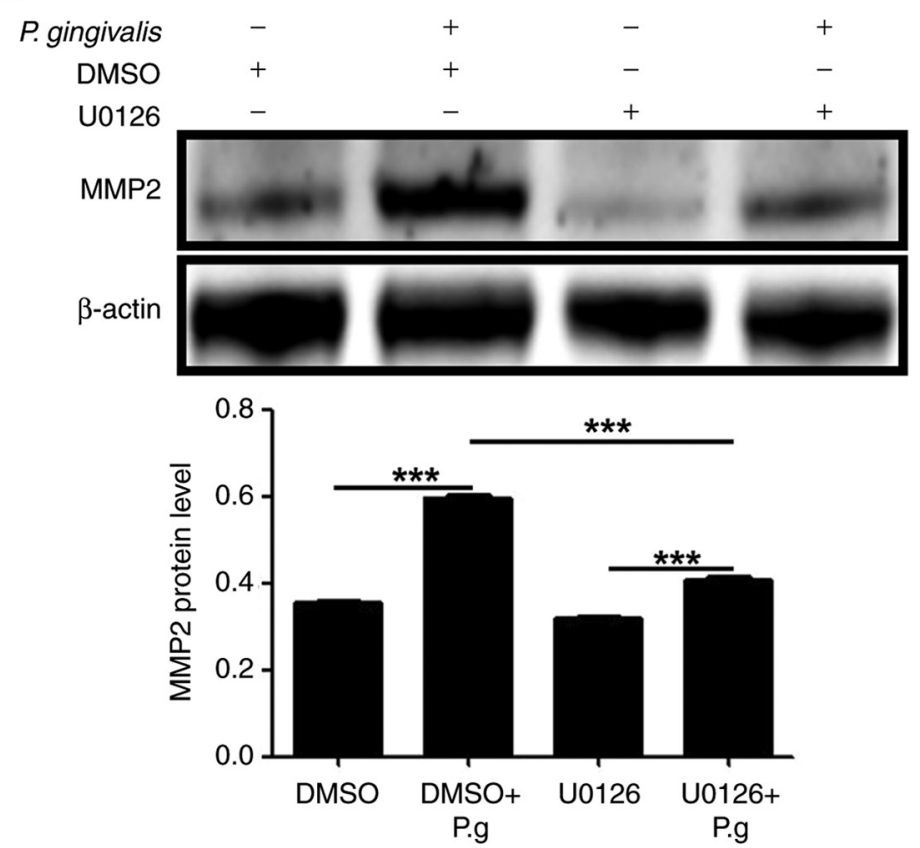

Figure 5. JNK and Erk1/2 are responsible for $P$. gingivalis induced MMP2 production. The upregulated expression of MMP2 at the (A) mRNA and (B) protein level in human macrophages infected $P$. gingivalis bacteria decreased when pre-treated with the JNK inhibitor before infection. The upregulated MMP2 (C) mRNA and (D) protein levels in response to infection with live $P$. gingivalis bacteria decreased when pre-treated with the Erk1/2 inhibitor. ${ }^{*} \mathrm{P}<0.05$, ${ }^{* *} \mathrm{P}<0.01,{ }^{* * *} \mathrm{P}<0.001$. ns, not significant; MMP2, matrix metalloproteinase 2; P. gingivalis, Porphyromonas gingivalis; IgG, immunoglobulin; Ab, antibody.

identified MMP2 and TNF- $\alpha$ as novel biomarkers of inflammation in the alveolar bone and neighboring tissues of patients with chronic periodontitis. Fu et al (17) found that cyclosporin-A prevented periodontal bone loss in patients with periodontitis by suppressing the expression of MMP2 and MMP9. Araújo et al (18) found that the inflammation and bone loss in patients with periodontitis was reduced when treated with olmesartan by decreasing MMP2, MMP9 and RANKL expression, and increasing osteoprotegerin production. In the present study, it was shown that the expression of MMP2 was upregulated in the PICF of patients with peri-implantitis. This observation is consistent with a report showing that active MMP2 complexes contributed to periodontal destruction and was found at higher levels in the periodontal ligament and gingival crevicular fluid of patients with periodontitis (19).

Osteoclastogenesis and severe macrophage derived inflammatory responses can cause peri-implantitis (20). Thus, human macrophages were selected for infection by $P$. gingivalis to assess the regulatory mechanism of MMP2 in peri-implantitis. 
Kong et al (21) revealed that the secretion of MMP1 and MMP2 induced by $P$. gingivalis stimulation was significantly inhibited by theaflavins in human gingival fibroblasts. Mieszkowska et al (22) found that an increase of MMP2, IL-1 $\beta$ and IL-8 following infection with $P$. gingivalis was decreased by rhamnogalacturonan-Is treatment, which can reduce inflammation and enhance implant integration. Consistent with these studies, the results of the present study showed that when cells were infected with $P$. gingivalis, MMP2 levels were increased in the normal human macrophages.

LOX-1 and dectin-1, as pattern recognition receptors of the C-type lectin superfamily, are associated with osteoclastogenesis (23-25). Bacteria, fungi, apoptotic cells and oxidized low-density lipoproteins are the primary ligands of LOX-1 $(26,27)$. Nakayachi et al (23) found that osteoclast differentiation could potentially be inhibited by decreasing the cell-cell fusion of preosteoclasts via LOX-1. Dectin-1, found predominantly on the cell membrane of the cells of a myeloid lineage, can recognize $\beta$-glucans, some bacteria and other unidentified molecules (28). Yamasaki et al (24) found that osteoclast differentiation and NFATc1 expression could be negatively regulated by the dectin-1/syk signaling pathway. In the present study, the data showed that MMP2 production was regulated by LOX-1 in human macrophages with $P$. gingivalis. The results of the present study are in agreement with previous studies that have shown that LOX-1 neutralizing antibody treatment can reduce MMP2 and MMP9 expression induced by gasoline and diesel engine exhaust (29). The results of the present study also revealed that MMP2 production was independent of dectin-1 in infected human macrophages with $P$. gingivalis.

JNK and Erk1/2, as members of the serine-threonine protein kinases, play significant roles in cellular responses to proinflammatory cytokines. Li et al (30) found that the expression of IL-1 $\beta$ and TNF- $\alpha$ induced by sialidase of $P$. gingivalis could be inhibited by JNK blocking epi 4 cells. Ren et al (31) found that $P$. gingivalis induced IFN- $\gamma$ and IL-8 expression was mediated by Erk1/2 and p38 MAPK in HTR 8 cells. JNK and Erk1/2 are also involved in MMP2 secretion in inflammatory diseases. Jiang et al (32) showed that neuropathic pain induced by chronic constrictive injury was significantly attenuated by tetramethylpyrazine via the JNK-MMP2/9 signaling pathway. Wang et al (33) found that Erk1/2/NF- $\kappa$ B pathway could upregulate MMP2 and MMP9 expression in murine mast cells infected with Toxoplasma gondii. The results of the present study suggested that JNK and Erk1/2 were responsible for $P$. gingivalis induced MMP2 production. The Erk1/2 inhibitor was considerably more potent than the JNK inhibitor in suppressing MMP2 production.

In conclusion, the results of the present study, obtained through evidence from patients and cell-culture studies, showed that MMP2 was involved in peri-implantitis, and may have been regulated by LOX-1, in a JNK and Erk1/2 signaling pathway dependent manner. The LOX-1/MMP2 signaling pathway may thus be a potential target for management of peri-implantitis.

\section{Acknowledgements}

Not applicable.

\section{Funding}

The present study was sponsored by the Traditional Chinese Medicine Research Project of Qingdao (grant no. 2020-zyy055) and the Science and Technology Project of Qingdao West Coast New District (grant no. 2020-54).

\section{Availability of data and materials}

The datasets used and/or analyzed during the present study are available from the corresponding author on reasonable request.

\section{Authors' contributions}

QZ designed the study and wrote the majority of the manuscript. TX and NB performed the majority of the experiments and wrote part of the manuscript. FT and HZ performed some of the experiments. JL designed the study and revised the manuscript. All authors have read and approved the final manuscript. QZ and TX confirm the authenticity of all the raw data.

\section{Ethics approval and consent to participate}

The present study was approved by the Ethics Committee of The Affiliated Hospital of Qingdao University (Qingdao, China) and performed in accordance with the Helsinki Declaration. Each patient in this study provided written informed consent.

\section{Patient consent for publication}

Not applicable.

\section{Competing interests}

The authors declare that they have no competing interests.

\section{References}

1. Zhang Q, Xu H, Bai N, Tan F, Xu H and Liu J: Matrix metalloproteinase 9 is regulated by LOX-1 and erk $1 / 2$ pathway in dental peri-implantitis. Curr Pharm Biotechnol 21: 862-871, 2020.

2. Zhang Q, Liu J, Ma L, Bai $\mathrm{N}$ and Xu H: LOX-1 is involved in TLR2 induced RANKL regulation in peri-implantitis. Int Immunopharmacol 77: 105956, 2019.

3. Roccuzzo A, Stähli A, Monje A, Sculean A and Salvi GE: Peri-implantitis: A clinical update on prevalence and surgical treatment outcomes. J Clin Med 10: 1107, 2021.

4. Wang Y, Zhang Y and Miron RJ: Health, maintenance, and recovery of soft tissues around implants. Clin Implant Dent Relat Res 18: 618-634, 2016.

5. Barootchi S and Wang HL: Peri-implant diseases: Current understanding and management. Int J Oral Implantol (Berl) 14: 263-282, 2021.

6. Corry DB, Rishi K, Kanellis J, Kiss A, Song LZ, Xu J, Feng L, Werb Z and Kheradmand F: Decreased allergic lung inflammatory cell egression and increased susceptibility to asphyxiation in MMP2-deficiency. Nat Immunol 3: 347-353, 2002.

7. Puliti M, Momi S, Falcinelli E, Gresele P, Bistoni F and Tissi L: Contribution of matrix metalloproteinase 2 to joint destruction in group B Streptococcus-induced murine arthritis. Arthritis Rheum 64: 1089-1097, 2012.

8. Lu CY and Lai SC: Induction of matrix metalloproteinase-2 and -9 via Erk1/2-NF- $\mathrm{KB}$ pathway in human astroglia infected with Toxoplasma gondii. Acta Trop 127: 14-20, 2013. 
9. Barreiros D, Filho NP, Paula-Silva FWG, Oliveira KMH, Lucisano MP, Rossi AD, Silva LAB, Küchler EC and Silva RAB: MMP2 and MMP9 are associated with apical periodontitis progression and might be modulated by TLR2 and MyD88. Braz Dent J 29: 43-47, 2018.

10. Che C, Liu J, Ma L, Xu H, Bai N and Zhang Q: LOX-1 is involved in IL-1 $\beta$ production and extracellular matrix breakdown in dental peri-implantitis. Int Immunopharmacol 52: 127-135, 2017.

11. Park E, Na HS, Kim SM, Wallet S, Cha S and Chung J: Xylitol, an anticaries agent, exhibits potent inhibition of inflammatory responses in human THP-1-derived macrophages infected with Porphyromonas gingivalis. J Periodontol 85: e212-223, 2014.

12. Che C, Liu J, Ma L, Xu H, Bai N and Zhang Q: Osteopontin is essential for IL-1 $\beta$ production and apoptosis in peri-implantitis. Clin Implant Dent Relat Res 20: 384-392, 2018.

13. Zhao G,Hu M, Li C, Lee J, Yuan K, Zhu G and Che C: Osteopontin contributes to effective neutrophil recruitment, IL-1 $\beta$ production and apoptosis in Aspergillus fumigatus keratitis. Immunol Cell Biol 96: 401-412, 2018

14. Livak KJ and Schmittgen TD: Analysis of relative gene expression data using real-time quantitative PCR and the 2(-Delta Delta C(T)) method. Methods 25: 402-408, 2001.

15. Goncalves PF, Huang H, McAninley S, Alfant B, Harrison P, Aukhil I, Walker C and Shaddox LM: Periodontal treatment reduces matrix metalloproteinase levels in localized aggressive periodontitis. J Periodontol 84: 1801-1808, 2013.

16. da Costa T.A, Silva MJ, Alves PM, Chica JE, Barcelos EZ, Giani MA, Garlet GP, da Silva JS, Júnior VR, Rodrigues DB and Cardoso CR: Inflammation biomarkers of advanced disease in nongingival tissues of chronic periodontitis patients. Mediators Inflamm 2015: 983782, 2015 .

17. Fu MM, Fu E, Kuo PJ, Tu HP, Chin YT, Chiang CY and Chiu HC: Gelatinases and extracellular matrix metalloproteinase inducer are associated with cyclosporin-A-induced attenuation of periodontal degradation in rats. J Periodontol 86: 82-90, 2015.

18. Araújo AA, de Souza GL, Souza TO, de Castro BritoGA, Aragão KS, de Medeiros CA, Lourenço Y, Alves MS and de Araújo RM Jr: Olmesartan decreases IL-1 $\beta$ and TNF- $\alpha$ levels; downregulates MMP-2, MMP-9, COX-2, and RANKL; and upregulates OPG in experimental periodontitis. Naunyn Schmiedebergs Arch Pharmacol 386: 875-884, 2013.

19. Bildt MM, Bloemen M, Kuijpers-Jagtman AM and Hoff JW: Collagenolytic fragments and active gelatinase complexes in periodontitis. J Periodontol 79: 1704-1711, 2008.

20. Tang H, Mattheos N, Yao Y, Jia Y, Ma L and Gong P: In vivo osteoprotegerin gene therapy preventing bone loss induced by periodontitis. J Periodontal Res 50: 434-443, 2015.

21. Kong L, Qi X, Huang S, Chen S, Wu Y and Zhao L: Theaflavins inhibit pathogenic properties of $\mathrm{P}$. gingivalis and MMPs production in P. gingivalis-stimulated human gingival fibroblasts. Arch Oral Biol 60: 12-22, 2015.
22. Mieszkowska A, Folkert J, Gaber T, Miksch K and Gurzawska K Pectin nanocoating reduces proinflammatory fibroblast response to bacteria. J Biomed Mater Res A 105: 3475-3481, 2017.

23. Nakayachi M, Ito J, Hayashida C, Ohyama Y, Kakino A, Okayasu M, Sato T, Ogasawara T, Kaneda T, Suda N, et al: Lectin-like oxidized low-density lipoprotein receptor-1 abrogation causes resistance to inflammatory bone destruction in mice, despite promoting osteoclastogenesis in the steady state. Bone 75: 170-182, 2015

24. Yamasaki T, Ariyoshi W, Okinaga T, Adachi Y, Hosokawa R, Mochizuki S, Sakurai K and Nishihara T: The dectin 1 agonist curdlan regulates osteoclastogenesis by inhibiting nuclear factor of activated T cells cytoplasmic 1 (NFATc1) through syk kinase. J Biol Chem 289: 19191-19203, 2014.

25. Yang H, Wang Q, Han L, Yang X, Zhao W, Lyu L, Wang L, Yan $\mathrm{H}$ and Che C: Nerolidol inhibits the LOX-1/IL-1 $\beta$ signaling to protect against the Aspergillus fumigatus keratitis inflammation damage to the cornea. Int Immunopharmacol 80: 106118, 2020.

26. Cheng M, Lin J, Li C, Zhao W, Yang H, Lv L and Che C: Wedelolactone suppresses IL-1 $\beta$ maturation and neutrophil infiltration in Aspergillus fumigatus keratitis. Int Immunopharmacol 73: 17-22, 2019.

27. Che C, Li C, Lin J, Zhang J, Jiang N, Yuan K and Zhao G: Wnt5a contributes to dectin-1 and LOX-1 induced host inflammatory response signature in Aspergillus fumigatus keratitis. Cell Signal 52: 103-111, 2018.

28. Drummond RA and Brown GD: The role of dectin-1 in the host defence against fungal infections. Curr Opin Microbiol 14: 392-399, 2011.

29. Wu Z, Sawamura T, Kurdowska AK, Ji HL, Idell S and Fu J: LOX-1 deletion improves neutrophil responses, enhances bacterial clearance, and reduces lung injury in a murine polymicrobial sepsis model. Infect Immun 79: 2865-2870, 2011

30. Li C, Yang X, Pan Y, Yu N, Xu X, Tong T, Tang X, Zhang D, Liu J and Lin L: A sialidase-deficient porphyromonas gingivalis mutant strain induces less interleukin-1 $\beta$ and tumor necrosis factor- $\alpha$ in Epi4 cells than W83 strain through regulation of c-Jun N-terminal kinase pathway. J Periodontol 88: e129-e139, 2017.

31. Ren H, Li Y, Jiang H and Du M: Porphyromonas gingivalis induces IL-8 and IFN-gamma secretion and apoptosis in human extravillous trophoblast derived HTR8/SVneo cells via activation of ERK1/2 and p38 signaling pathways. Placenta 45: 8-15, 2016.

32. Jiang L, Pan CL, Wang CY, Liu BQ, Han Y, Hu L, Liu L, Yang Y, Qu JW and Liu WT: Selective suppression of the JNK-MMP2/9 signal pathway by tetramethylpyrazine attenuates neuropathic pain in rats. J Neuroinflammation 14: 174, 2017.

33. Wang MF, Lu CY and Lai SC: Up-regulation of matrix metalloproteinases-2 and -9 via an Erk1/2/NF- $\kappa$ B pathway in murine mast cells infected with Toxoplasma gondii. J Comp Pathol 149: 146-155, 2013. 